\title{
AI Fitness Checks, Maintenance and Monitoring on Systems Managing Content \& Data: A Study on CMS World
}

\author{
Apoorva Ganapathy
}

Senior Developer, Adobe Systems, San Jose, California, USA

\begin{abstract}
Artificial intelligence health check, monitoring, and maintenance on system managing content in the CMS world are essential to prevent damage and avoidable expenses. To also avoid loss of time from massive downtime, AI has been employed for maintenance functions. Servers which are the houses and homes for data and content from computers, have to be maintained to allow the system to function at an optimal level. The use of AI for this maintenance involves a lot of factors. This work discusses these factors and how the various aspects work to monitor and maintain systems managing content and data. AI is programmed using health monitoring scripts which are commands executed by a particular programming language. This scripts program the AI to check and monitor servers for maintenance function. This maintenance aims to track CPU optimization and allow for the best functioning of the CPU using preventive and predictive maintenance techniques.
\end{abstract}

Key words: Servers Maintenance, Artificial Intelligence (AI), Automated Machine, Repository Optimization, Data Script, Central Processing Unit (CPU)

$12 / 31 / 2015$

This article is is licensed under a Creative Commons Attribution-NonCommercial 4.0 International License. Attribution-NonCommercial (CC BY-NC) license lets others remix, tweak, and build upon work non-commercially, and although the new works must also
acknowledge \& be non-commercial.

\section{INTRODUCTION}

Servers help in storing data. They also help improve productivity and efficiency; this is because data stored on them can be retrieved from any place and on the go. Significant companies with immense data and content depend on servers to allow for communication. Sometimes they struggle with keeping the server up and running $24 / 7$. Whenever the server doesn't work at the expected level, the data that the companies depend on for the execution of critical tasks won't get to the intended destination on time or sometimes at all. It becomes vital to monitor, maintain and check the health of the server from time to time. Several tools can be used to this, including manual checkup and maintenance. These tools are artificial intelligence tools (Automated). They ensure that servers, both physical and virtual, are working and functioning at an optimal level for best results. Servers need to perform at the best levels. Failure of a server can have a severe effect on the business of the company because servers are where all the companies' data is stored. When a server fails, it will affect the delivery of the business services. It is therefore imperative to use health check to examine any performance problems so that they can be identified as early as possible.

\section{SERVER}

A server compromises similar parts to your typical desktop computer system. That is it as a processing chip, memory, motherboard, etc. However, the components and makeup of a server are more complex and design to conduct more exhausting operations. For example, servers usually have more significant storage and memory capacity compared to everyday computers. This is because they have larger RAM (Read Access Memory). To have more memory capacity would require using better quality parts in supporting the high storage capacity. Servers are designed to allow for the highest computing power while consuming the smallest space possible. The purpose of the extra power and ability for servers is for the workload. Servers are created to process data on request and give back the required suiting information. The information may be sent back to the actual requester. It may also be sent to other computers through a local network or through the internet.

Servers are access points on various networks connected through a local network or the internet. It has to be connected to some network that can enable users to reach the servers and collect the information stored on them. The whole point of having a server is keeping data and allowing users access to the data, so the users must access the stored data. If you use the internet, your servers, and when you know it, they are now part of our typical everyday life. 
For every site (URL) you visit through your browser, you send a request to the site's server. At this point, there is a request, and the server processes that request and answers the request through your computer by giving it the right information and direction to the site. This is referred to as the client-server model. This means that the device (computer, phone, tablet, etc.) is the client who is asking for the information; the server where the information is stored is just a storage unit that provides you information. Also, aside from being used for storing internet information, servers may be used to connect different computers using local networks (Movva et al., 2012). This usually occurs in workplaces. For instance, after creating a username and password which allows you to login into any computer at work, your information is stored in your office servers. This will enable you to save files (pictures, documents, videos) on a particular computer and still be able to open them on different computers. Here, computers in your workplace are connected to the server through a local network, and the information stored on the server can be accessed through the connected computers.

There are different types of servers designed to provide specific services. They are usually arranged in groups. Since servers are designed for particular purposes and uses, each server does one type of job. This makes it challenging to have a single server. Instead, you will usually notice a server room with many servers all put up and arranged orderly in what looks like a vast library called racks. Big companies install several servers to boost and generate enough processing power to accommodate different systems, applications, and databases.

There are also virtual servers (server virtualization) that connect ware (hardware or software) with other operating systems. Virtual servers are more cost and energy-efficient because they share hardware and software. In Server virtualization, software applications are applied to a physical Server dividing the primary server into multiple virtual servers. The main server remains the actual server that brings the inception of several servers. So you have a single server with several virtual servers stored within it. An advantage of this is that the virtual servers can be accessed directly through your computer instead of remotely and physically. Servers play a crucial role in our everyday modern world where the internet is used daily. They store all the information we need and use every day.

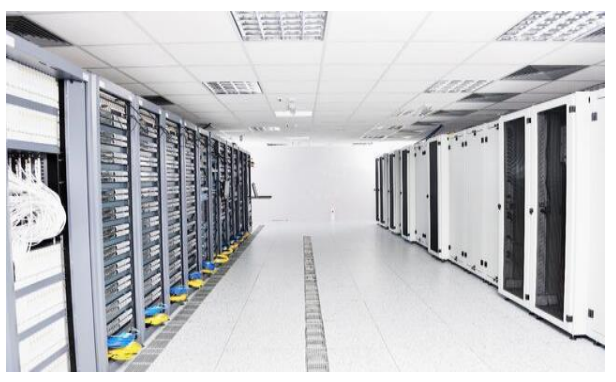

Figure 1: Server room (Source: https://blog.etech7.com/)

\section{Server Health Maintenance and Monitoring}

Servers have to be maintained for performance best and to prevent breakdown. Maintenance is the process of ensuring that the server is updated and working at an optimal level.It is vital to maintain and look after your servers because they house and take care of all your data. This is to ensure that you don't lose valuable data in the event of damage. The maintenance would include constant monitoring and continuously analyzing the way the server functions. This would allow you to know right away if something is up with the server and give you the chance to fix the issue quickly before it gets worse. In addition to noticing present needs, server maintenance also includes preemptive measures. There is a need to keep your servers safe and updated before it goes really bad. Preemptive maintenance reduces future server issues as well as arranges your network should something go wrong.

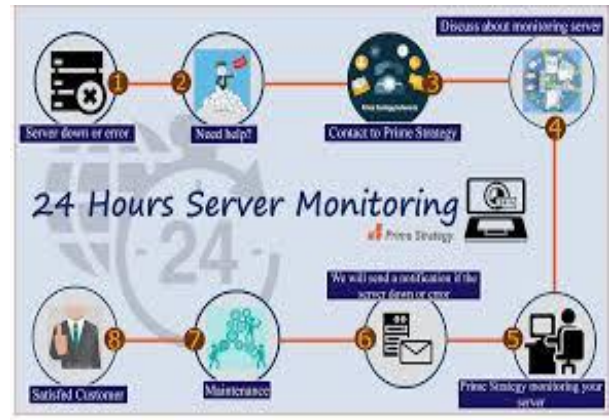

Figure 2: Server monitoring system (Source: $\underline{\text { www.prime-strategy.co.id) }}$ 


\section{IMPORTANCE OF SERVER MAINTENANCE}

It is essential to know that server maintenance involves virtual and physical care. At the same time, maintenance involving software updates and backups (virtual configuration) may be obvious, physical maintenance can often be disregarded. It is, however, equally important. Servers need to be cleaned and dust to allow for ventilation. Servers need a proper temperature and good room conditioning. Servers should be kept in a cool place.

Server maintenance is like putting gas in your car. You shouldn't run out of gas entirely before you buy some more because, at that point, you won't be able to drive. You need to think ahead and fill up your car as you need for your trips. Maintaining your server is quite the same. You need to take care of your server before it gets really bad. Regular server maintenance is essential for companies (big or small). As long as you use a server, there is a need to maintain it to keep it at an optimal and reliable level. Taking good care of your server will help increase its life span while saving time and money. Maintenance will spare you from having to buy a new server. It will also save you time and increase efficiency. It is about staying proactive and, above all, issues ahead of the game. Specific importance of server maintenance includes:

- Keeping the Operating System (OS) updated: Server OS updates are released from time to time. These updates are usually improvements from the last update. Security-wise, hackers look through systems for vulnerabilities and get to know the easiest to attack and how. With regular maintenance, the OS would be updated to the best version to avoid hacking. For this reason, an automated management and monitoring system is essential. An AI will inform you of the latest update and allow you to update automatically.

- Checking Resource Utilization: Frequent maintenance will allow you to review server RAM, CPU, and disk utilization. You will also find out whether or not the System has been stretched to its limits.

- Physical cleaning: Through routine maintenance, servers would be cleaned. Servers may collect dust that clogs the ventilation points. This has to be done through manually cleaning and removing from time to time.

- Update web applications: Web applications create the majority of the security breaches witnessed. Web apps are more prone to Internet attacks such as installation malware and viruses since they are the main connectors to the internet. Conducting frequent web application updates will allow for proper security.

- Backup verification: This task is essential to server maintenance. Backups must be frequent (daily or weekly) depending on the volume of data generated by the users.

\section{Al Health Monitoring and Maintenance}

Server maintenances and monitoring are not new. They've been taking place almost since computing, and the essence is to detect and solve any possible problem. Things have come a long way from the rigid, prescriptive servicing method, which may be unable to detect possible issues and cause very costly and expensive machine damage or even complete loss. Enter the era of Artificial Intelligence (AI) maintenance which includes several techniques used by automated and programmed machines to maintain servers. Using AI for system managing content and data on CMS will help put everyone in the maintenance game. That is, both automated machine operators and actual technicians will be involved in system maintenance. By having AI perform regular checks and monitoring, the workload on the technician will be reduced, and they will have to pay attention to more enormous changes for improvement in the reliability of the machines. The fast emergence of AI-powered technologies in predicting faults (preventive and predicting maintenance) takes a new lead. AI maintenance check jobs on systems managing content and data in the Content management world is becoming more intelligent and moving into a far more advanced stage in the area of the range of activities that can be monitored, checked and the pace at which data and information originating from one computer may be analyzed \& examined for taking efficient and effective actions. Using AI for maintenance can monitor analytics to detect machine anomalies often before actual issues or unexpected events happen. For example, machine learning now allows enterprises to High level of real-time maintenance of different factors, such as audio, temperature, and other miscellaneous and extensive aspects of production machinery (Vadlamudi, 2015). Huge industries with a big server base, there has been an increased attention on AI-powered predictive maintenance with highly advanced applications detecting and solving machine irregularities caused by wear and tear from the day to day usage. A large number of servers, computers, and machines maybe supervised and monitored. The maintenance criteria detected in line with the status of single components. Through machine learning, AI-powered predictive maintenance can provide an increased understanding of deviations by machines from normal behavior. AI maintenance can identify failures and give signals many months in advance. Action planning algorithms are usually added on by machine learning to identify failures in time.

The machine learns and develops from its own experience using deep learning neural networks. The consistent use of automated and artificial intelligence for maintenance provides a speedy and thorough real-time machine condition consciousness. Data storing, examining and processing in large amount is essential for automated machines. However, 
they have to promote advanced and highly advanced graphics for picture processing. They must also be easy, quick to configure, and highly efficient to withstand the usually tasking and complex environment where it operates. AI maintenance tries to reduce the potential of failure. This it does by detecting changes or anomalies and tries to fix it before it becomes an even bigger problem. It also finds connecting relationships in data, analyses available data, detects where the error is and sets up techniques to alter them. This is what preventive maintenance is all about. AI monitoring will help companies manage and maintain their repository using data. This data shall help with the maintenance schedule to reduce system crash and avoid failure. AI is often tricky and tasking to install. It would be best if you had a lot of communication and training to implement an AI into your maintenance system. AI may not have all the said abilities that human technicians do because they might lack historical computer knowledge and are based on their script. They have now automated machine-driven software, which allows server management and maintenance simple. Machine learning and sensor data model make it possible to quickly get extra value from the colossal volume of distorted data. Predictive system management tools also upgrade available maintenance system through the use of AI to make sure that the correct information and tools needed to maintain critical assets needed for the execution of the task at the best levels.

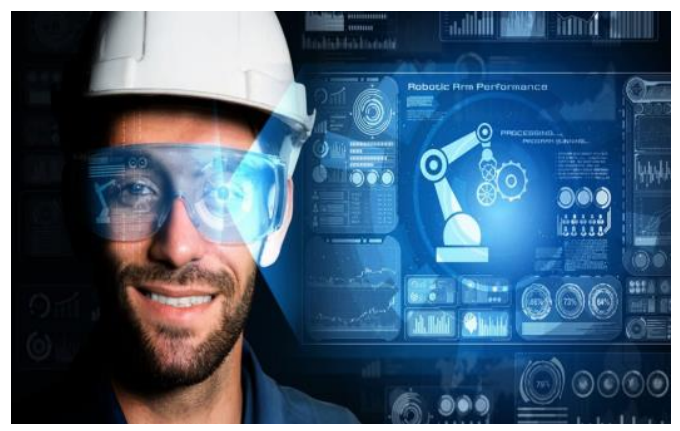

Figure 3: Human vs Machine maintenance (Source: https://dlabs.ai/blog)

\section{Al Monitoring and Management System over Traditional Human Sever System Checks}

The checking of servers and computer system has been in existence for as long as we have had computers. The essence of server checks is to prevent system downtime and reduced productivity while increasing uptime through preventive measures and monitoring. The traditional method involves technicians routinely inspecting the hardware or software of servers to make sure that all functioning is working according to the normal functions. It also captures possible faults and signs of hardware issues. The frequency and extent to which the maintenance checks occur depend on the company and when they deem it fit. Regardless of the regularity, there could still be likely something going wrong or undetectable fault during and within checks. This brings Artificial Intelligence machine maintenance and monitoring into the picture. They can report the fault and potential system failure (Ujwala et al., 2012). Through AI monitoring, companies using servers and are in a place that needs considerable availability; it would be a disaster should the content management and server went down for any duration. One-time signals, notice, and warning of possible system crash will be of great advantage. On this, different algorithms have been used to offer maintenance services that monitor and checks not just key metrics (CPU, storage and memory utilization) but also provide analyzed result of software update status, security breaches, antivirus patch, and a lot of other system health check attributes. AI check may automatically act on application updates, automate server, and future server scheduled restarts to fit the environment. It also reduces the likelihood of system crash during an emergency. Advanced AI monitoring solutions can alert technicians when there is a potential failure and log ticket report, so the incident is recorded.

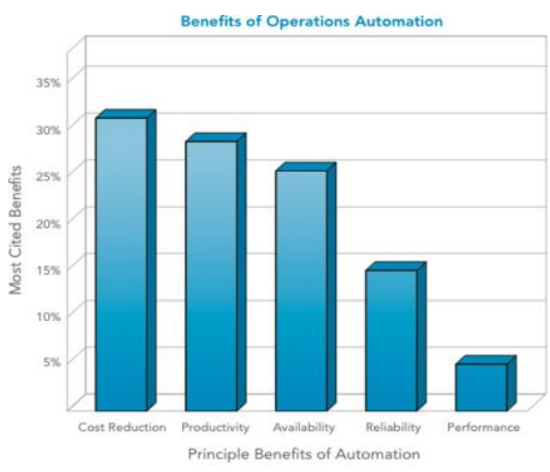

Figure 4: Benefits of automated maintenance (Source: Source: www.helpsystems.com) 


\section{Repository MaintenANCE}

Repository maintenance involves activities such as backing up available data and application settings. It also includes cleaning up unused and old data to ensure data integrity and peak performance.

Over time, servers tend to get larger. New data are entered, stored, saved, and they also accumulate. As a result of this inflow of further information, the repository database grows to a level that negatively affects the server's performance. The reduced performance may also slow down the time it takes to save a file. Some operations may take longer than usual in the worst-case scenarios to start or even fail with a timeout error. To prevent such problems, the removal of long untouched and unused data should be done.

Data that should be removed includes

- Expired data.

- Unnecessary data

- Old and unused data

\section{Ways of Maintaining Repository Data}

Deleting unwanted data may be done in several ways. You can remove them manually. Also, automated utility clean-up can be done using batch deletion of items that fit the criteria that you want deleted. Also, an AIs are programmed for scheduled automated deletion at regular intervals to prevent the impact of the enormous data deletion from negatively affecting the system's performance; a maintenance service manages the actual deletion.

- Repository Backups: Repository data can also be backed up. This involves storing the repository data and application settings in a relational database. Automated maintenance service removes flagged items periodically to optimize system performance.

- Batch Deletion: Deleting a large amount of data can be extremely tedious if you do them separately, and you need to add each separately. Where the items.

\section{HeALth Monitoring ScRiPtS}

These are program commands worked on through particular script engines. Scripts may be used for diverse functions on a system. Script prepare artificial intelligence pattern for a local network. It develops web pages for the web. A script could process operating systems to control functions on them. Different script Languages exist along with scripting files. Script files hold commands written down in a specific programming language. Scripts are files, and they can be stored. This means that they may also be accessed, adjusted, and edited to alter the initial commands. CPU functions and utilization differs. CPU utilization involves the way computers use their processing powers or resources and the CPU's job performance. Various computer utilization differs depending on the computer processor, the types and amount of computing jobs managed by the CPU. Some jobs may require enormous CPU time (Narayana et al., 2012). Other tasks may not necessarily need much CPU time. There are scripts available to monitor the health status of web servers and services. The scripts are installed and configured for maintenance checks, monitoring, and reporting. They also provide many advanced features that monitor things on your server. Health scripts are simple solutions for basic health checks on your web resources. They can also keep track of server processor functions and utilization. CPU utilization means a computer's usage of the processing resources (usage of the RAM). It could also mean the quantity of work handled. The CPU utilization of computers varies depending on the computing jobs and the workload. Different jobs require a large amount of CPU time. Some CPU workload is small, and lights and therefore requires fewer CPU resources. AI health monitoring scripts monitor CPU utilization and instance up a time to manage to be running forever by having a repository and clean up jobs run during the server uptime period.

\section{Top Server Maintenance Software}

- SolarWinds server and application Monitor (SAM): this monitors Dell PowerEdge, IBM eServer, xSeries, HP Proliant, Microsoft Servers and various others.

- Atera; this management to I'd cloud-based. It manages networks and servers. That is, maintenance of application features, connectivity of local networks and servers.

- ManageEngine OpManager: This provides good management and maintenance for networks and servers. It consists of performance analysis for the various notable server resource metrics. It is available for Linux ad windows server. 
- Site24x7 server: this is also a maintenance tool based on a cloud system. It monitors a range of servers. They include Linux and other window-based Servers.

- SolarWinds RMM: this maintenance tool allows operators to work outside the usual management system. It manages and monitors the server also.

- Checkmark: This maintenance tool performed servers, network and application maintenance both onsite and in the cloud.

- Syxsense manage: this is a cloud-based service that can maintain servers from any location. It also manages endpoints—-syxsense groups its supervision into one main overview.

- ManageEngine Applications Manager: this tool covers a large variety of functions. It monitors the server's health and gives live status updates on computer utilization, the disk capacity of storage, and the level of uptime.

- Paessler PRTG Network Monitor: This maintenance tool unifies several maintenance features such as network, server and application Monitor. It also supervises CPU performance and capacity.

- Server Density: it performs most maintenance functions o AI including power supply health check of servers. It is also both a cloud-based and direct maintenance tool.

\section{Al in The Content Management System (CMS)}

According to Patel, AI is likely to change the systems themselves. The following are few ways through which AI can change Content Management System (CMS)

- Real-Time SEO Recommendations: Think of CMS that can identify your content in real-time in search engine optimization. AI could suggest whether your content is better than your competitors in search engine optimization (SEO). It may pick out terms that you should or shouldn't use and also provide recommendations

- Advanced analytics, businesses grow and gain more customers: CMS's analytics will likely become more advanced and complex. Using history, content management systems will understand the entire user journey and may be able to foretell the type of content that leads to the most engagement and the highest likelihood of s conversation.

- Connection with other AI systems: It is common knowledge that voice-activated devices interact with other intelligent devices. At the rate of development across the AI space, it won't be long before CMS also begins to connect to these devices as well. With time AI will be able to optimize the user interface and content and the user experience for the best experience and satisfaction.

- Intelligent DAM: AI Haven has gone far into digital assets and content management systems except for automated image tagging and categorization. However, with the growth of AI, you will be able to search for content within videos, automatic and accurate voice-to-text transcription of the video, and so on.

\section{CONCLUSION}

The pace of development and growth of artificial intelligence in our world today cannot be overemphasized. Computers have become part of our daily and social life. This has made their use more necessary, and as more people use them, the need to maintain becomes even more essential. Maintenance would save them from preventable damages that may lead to loss of data. Though technicians (Human Beings) could perform maintenance checks, AI (Artificial Intelligence or Automated machines) have used for this maintenance. AI performs preventive and predictive maintenance, giving signals and warning of computer anomalies to enable the taking of the proper corrective actions. These maintenance strategies on servers and data management systems will help prevent data loss from server damage. They also optimize the performance of system functions and CPU utilization. Also, through machine learning, the AI can develop and advance in their maintenance and management of computers, detecting future anomalies and changes beforehand.

\section{REFERENCES}

Movva, L., Kurra, C., Koteswara Rao, G., Battula, R. B., Sridhar, M., \& Harish, P. (2012). Underwater Acoustic Sensor Networks: A Survey on MAC and Routing Protocols. International Journal of Computer Technology and Applications, 3(3).

Narayana, S. L., Suneetha Devi J., Bhargav Reddy I., Harish Paruchuri. (2012). Optimizing Voice Recognition using Various Techniques. CiiT International Journal of Digital Signal Processing, 4(4), 135-141.

Ujwala, D., Ram Kiran, D. S., Jyothi, B., Fathima, S. S., Paruchuri, H., Koushik, Y. M. S. R. (2012). A Parametric Study on Impedance Matching of A CPW Fed T-shaped UWB Antenna. International Journal of Soft Computing and Engineering, 2(2), $433-436$.

Vadlamudi, S. (2015). Enabling Trustworthiness in Artificial Intelligence - A Detailed Discussion. Engineering International, 3(2), 105-114. https://doi.org/10.18034/ei.v3i2.519

$$
--0--
$$

